\title{
A simple semiempirical method for predicting the temperature-entropy saturation curve of pure fluids
}

\author{
Juan A. White ${ }^{*, \dagger, \ddagger}$ and Santiago Velasco ${ }^{\dagger, \ddagger}$ \\ †Departamento de Física Aplicada, Universidad de Salamanca, 37008 Salamanca, Spain \\ $\ddagger$ Also at: IUFFyM, Universidad de Salamanca, 37008 Salamanca, Spain \\ E-mail: white@usal.es
}

\begin{abstract}
In this work we propose an approximate analytical method to obtain the liquidvapor saturation curve in a $T_{r}-s^{*}$ diagram, with $T_{r}=T / T_{c}, s^{*}=\left(s-s_{c}\right) / R, T_{c}$ the critical temperature, $s$ the molar entropy, $s_{c}$ the critical molar entropy and $R$ the gas constant, for a given fluid. The method uses a modified rectilinear diameter law for the saturated liquid and vapor entropies and an extended corresponding states equation for the entropy of vaporization. From this method two approximations are derived. The first approximation requires the use of data obtained from RefProp or a similar program. The second approximation only needs $T_{c}$, the critical molar volume, $v_{c}$, and the acentric factor, $\omega$, of the fluid as input data. For most fluids both approximations yield very good predictions.
\end{abstract}

\section{Introduction}

For about thirty years, organic Rankine cycles (ORC) have received increasing attention in the renewable energy sources research. An ORC works like a conventional Rankine cycle but 
uses an organic working fluid instead of water and it has been designed for producing electrical power from renewable energies (solar, geothermal, biomass) or from low-temperature waste heat, helping to reduce the consumption of fossil fuels.

The shape of the saturation liquid-vapor curve in a temperature-molar entropy $(T-s)$ diagram of a given fluid plays a crucial role in order to evaluate the possibility of its selection as the working fluid in an ORC. For all fluids, the saturation liquid-vapor curve in a $T-s$ diagram presents a more or less inclined forward bell or dome shape with two branches. The saturated liquid branch always has a positive slope while there are two possibilities for the slope of the saturated vapor branch: it is negative for any temperature between the triple and the critical point (wet fluids), or it can present a zone with positive values (dry fluids). Wet fluids always condense after the isentropic expansion in the turbine stage of a normal Rankine cycle without superheating while dry fluids may or may not condense depending on the condenser temperature. Usually, a third type of fluids is considered: isentropic fluids, with a nearly vertical saturated vapor branch. In this context White and Velasco ${ }^{1}$ have shown that the dry or wet character of ORC working fluids is well described by the maximum value attained by the slope of the saturated vapor branch in reduced coordinates. They have also shown that this maximum value is correlated with the critical molar volume, $v_{c}$.

Bao and Zhao $^{2}$ presented a review of the literature dealing with working fluid selections in an ORC, while Haervig et al. ${ }^{3}$ reported general guidelines about the optimal selection of working fluids for an ORC based on the temperature of the available heat source. Very recently, a novel classification scheme for pure working fluids in ORC has been reported by Györke et al. ${ }^{4}$ This classification scheme is based on the location of some characteristic points of the saturation liquid-vapor curve in a $T-s$ diagram.

The usual way of obtaining (screening) the saturation liquid-vapor curve in a $T-s$ diagram of a given fluid is the use of temperature and entropy data reported by a thermophysical property library. In this context, RefProp ${ }^{5}$ and CoolProp ${ }^{6}$ are two widely used libraries. RefProp is a non-open program with thermodynamics data for 147 fluids (in its 
most recent version RefProp 10.0) based on experimentally obtained equations of state (EoS). CoolProp is a recently available open-source program with similar capabilities. However, there are many fluids for ORC applications that do not appear in these libraries so that liquid-vapor coexistence data are not available from them.

An earlier attempt to describe the shape of the $T-s$ saturation boundary was made by Morrison in $1994^{7}$ in order to analyze the role played by this boundary in operating cycles in refrigerators and heat pumps. Morrison came to the conclusion that the important variation of the shape of the boundary from a working fluid to another is a consequence of molecular structure. Garrido et $a l .{ }^{8}$ performed a description of the geometry of the $T-s$ diagram of pure fluids based on different EoS. This study was latter extended to working mixtures in ORC. ${ }^{9}$ Very recently Groniewsky et al. ${ }^{10,11}$ have described the wet-to-dry transition in ORC working fluids by means of different model EoS. Finally, Su and coworkers ${ }^{12,13}$ have studied the slope of the vapor branch in a $T-s$ saturation curve by resorting to molecular groups techniques.

The aim of the present work is to provide approximate analytical equations for both the saturated liquid and the saturated vapor curves in a $T_{r}-s^{*}$ diagram (where $T_{r}$ is the reduced temperature $T / T_{c}$ and $s^{*}=\left(s-s_{c}\right) / R$, with $s_{c}$ the molar critical entropy and $R$ the gas constant) by using as input data only the critical temperature, $T_{c}$, the critical molar volume, $v_{c}$, and the acentric factor, $\omega$, of the fluid. We note that the use of the dimensionless molar entropy $s^{*}$ instead of $s$ allows for obtaining dimensionless results in a corresponding states principle (CSP) scheme without any loss of generality. The analysis is made from temperature and entropy liquid-vapor saturation data of the 121 fluids available by the National Institute of Standards and Technology (NIST) program RefProp $9.1^{5}$ (the most recent version RefProp 10.0 includes a larger number of pure fluids and new equations of state for some fluids but this should not affect the main conclusions of the present work). 


\section{Theory}

The method proposed in this work is based on the analysis of: 1) the temperature dependence of the lines of constant quality inside the liquid-vapor region of the $T_{r}-s^{*}$ diagram,

$$
s_{x}^{*}\left(T_{r}\right)=x s_{\mathrm{g}}^{*}\left(T_{r}\right)+(1-x) s_{1}^{*}\left(T_{r}\right)
$$

where $s_{\mathrm{g}}^{*}\left(T_{r}\right)$ and $s_{1}^{*}\left(T_{r}\right)$ are the saturated vapor and the saturated liquid entropies, respectively, and $x$ the quality of the liquid-vapor mixture; and 2) the temperature dependence of the entropy difference at saturation (the entropy of vaporization),

$$
\Delta_{\mathrm{v}} s^{*}\left(T_{r}\right)=s_{\mathrm{g}}^{*}\left(T_{r}\right)-s_{\mathrm{l}}^{*}\left(T_{r}\right)=\frac{\Delta_{\mathrm{v}} h_{r}}{T_{r}}
$$

where $\Delta_{\mathrm{v}} h_{r}=\Delta_{\mathrm{v}} h / R T_{c}$, with $\Delta_{\mathrm{v}} h$ the molar enthalpy of vaporization. From eqs 1 and 2 one has:

$$
\begin{gathered}
s_{\mathrm{g}}^{*}\left(T_{r}\right)=s_{x}^{*}\left(T_{r}\right)+(1-x) \frac{\Delta_{\mathrm{v}} h_{r}}{T_{r}}, \\
s_{1}^{*}\left(T_{r}\right)=s_{x}^{*}\left(T_{r}\right)-x \frac{\Delta_{\mathrm{v}} h_{r}}{T_{r}} .
\end{gathered}
$$

For most fluids, Bulavin et al. ${ }^{14}$ have shown that while the temperature dependence of the density diameter, $\rho_{d}=\left(\rho_{\mathrm{l}}+\rho_{\mathrm{g}}\right) / 2-\rho_{c}$, is close to linear in a wide temperature interval except very near the critical point (according with the so-called rectilinear diameter law), the behavior of $s_{d}^{*}\left(T_{r}\right)=s_{0.5}^{*}\left(T_{r}\right)$ is non-monotonous and sensitive to the behavior of the fluid. Therefore, using the entropy diameter $s_{d}^{*}\left(T_{r}\right)$, i.e., using $x=0.5$ in eq 1 , is not a good choice for getting general expressions for $s_{1}^{*}\left(T_{r}\right)$ and $s_{\mathrm{g}}^{*}\left(T_{r}\right)$. We have analyzed the temperature dependence of $s_{x}^{*}\left(T_{r}\right)$ for different values of the quality x. Figure 1 shows the liquid-vapor saturation curve in a $T_{\mathrm{r}}-s^{*}$ diagram together with the behavior of $s_{x}^{*}=s_{x}^{*}\left(T_{r}\right)$ for $x=0.2,0.4,0.6$ and 0.8 for the fluids $\mathrm{R} 32, \mathrm{RE} 143 \mathrm{a}$ and pentane $\left(\mathrm{C}_{5} \mathrm{H}_{12}\right)$ in the range $0.6<T_{r}<1$. In the three cases, $s_{x}^{*}=s_{x}^{*}\left(T_{r}\right)$ is practically linear for $x=0.4$. We have 

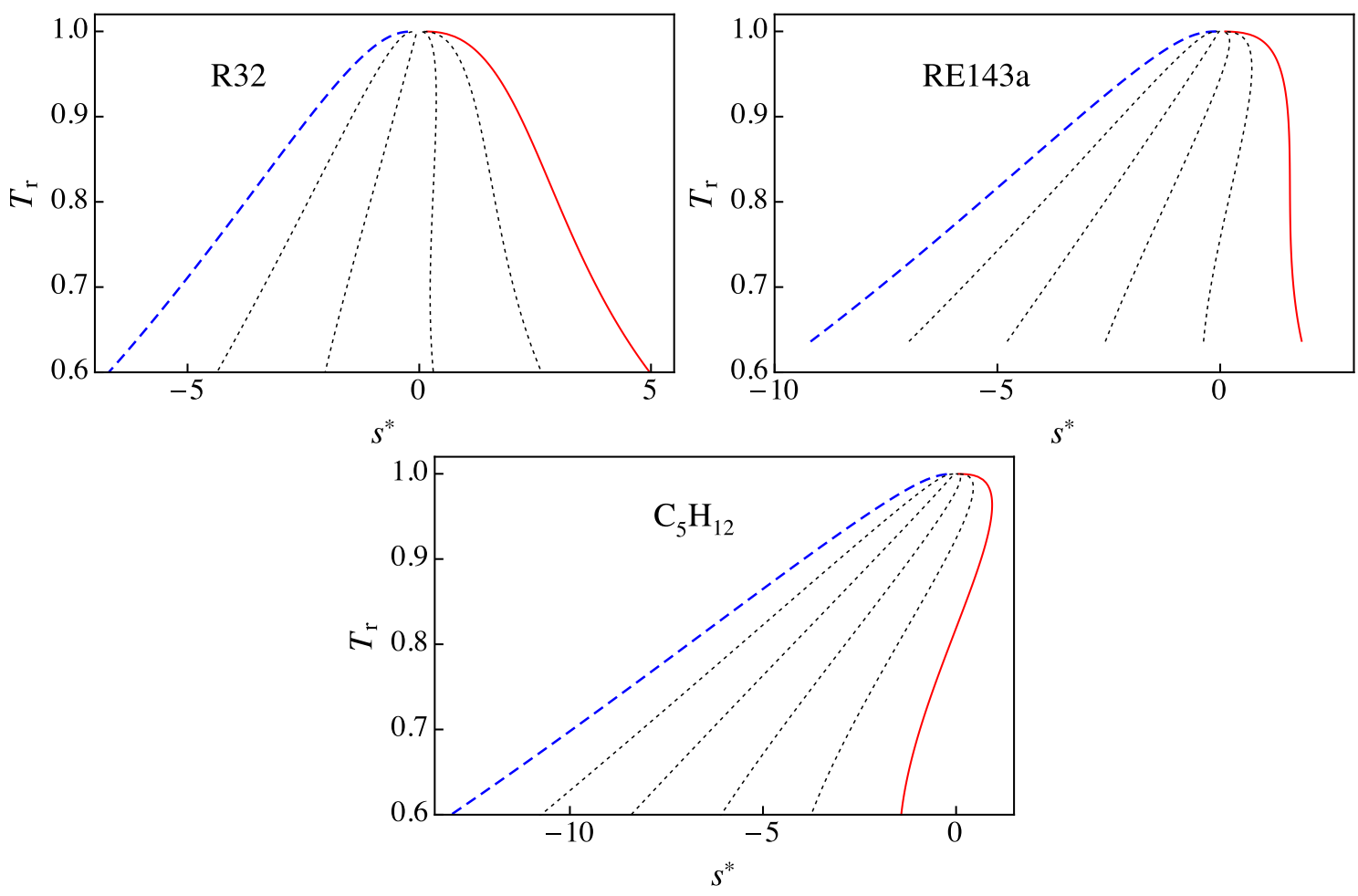

Figure 1: The liquid-vapor saturation curve in a $T_{\mathrm{r}}-s^{*}$ diagram for R32, RE143a and pentane. The dashed blue lines correspond to the liquid saturated branch while the solid red lines correspond to the vapor saturated branch. The dotted lines represent different values of the quality $x$ : from left to right $x=0.2,0.4,0.6$ and 0.8 . All data have been obtained from RefProp 9.1 results. ${ }^{5}$

checked this linear behavior for the fluids included in the RefProp 9.1 program. In particular, searching for the value of $x$ for which $s_{x}^{*}\left(T_{r}\right)$ reaches the best linear temperature dependence for all considered fluids, one obtains a mean value of $x=0.385$, so that

$$
s_{0.385}^{*}\left(T_{r}\right)=0.385 s_{\mathrm{g}}^{*}\left(T_{r}\right)+0.615 s_{1}^{*}\left(T_{r}\right) \approx a\left(1-T_{r}\right) \quad\left(0.6<T_{r}<1\right),
$$

where the coefficient $a$ is fluid dependent and gives the slope of the straight line. Eq 5 is strictly phenomenological and acts as an effective approximation not valid in the close neighborhood of the critical point $\left(0.99 \lesssim T_{r}<1\right)$. In relation with this fact, we note that Imre et $a l .{ }^{15}$ have recently observed the appearance of a small kink very close to the critical point in the two-phase isentrope ending in the critical point in a $T-x$ diagram. Equation 
5 plays the role of a modified rectilinear diameter law for the saturation entropies.

On the other hand, Velasco et al. ${ }^{16}$ have recently proposed the equation

$$
\Delta_{\mathrm{v}} h_{r}=B(\omega)\left(1-T_{r}\right)^{0.38}
$$

with

$$
B(\omega)=7.2729+10.4962 \omega+0.6061 \omega^{2} .
$$

for providing the enthalpy of vaporization, $\Delta_{\mathrm{v}} h_{r}$, in terms of the reduced temperature $T_{r}$ and the acentric factor $\omega$. An analysis of the performance of eqs 6 and 7 is provided by ref 16. By substituting eqs 5 and 6 into eqs 3 and 4, one obtains,

$$
\begin{aligned}
& s_{\mathrm{g}}^{*}\left(T_{r}\right)=a\left(1-T_{r}\right)+0.615 B(\omega) \frac{\left(1-T_{r}\right)^{0.38}}{T_{r}}, \\
& s_{1}^{*}\left(T_{r}\right)=a\left(1-T_{r}\right)-0.385 B(\omega) \frac{\left(1-T_{r}\right)^{0.38}}{T_{r}},
\end{aligned}
$$

with $B(\omega)$ given by eq 7 .

At this point it is convenient to recall that the only unknown parameter for determining $s_{\mathrm{g}}^{*}\left(T_{r}\right)$ and $s_{1}^{*}\left(T_{r}\right)$ is the slope $a$. We have checked that using $a$ from the fit of $s_{0.385}^{*}\left(T_{r}\right)$ data for a given fluid yields very good results for $s_{\mathrm{g}}^{*}\left(T_{r}\right)$ and $s_{1}^{*}\left(T_{r}\right)$ for $T_{r} \geq 0.6$. However, our goal is to obtain the temperature dependence of $s_{\mathrm{g}}^{*}\left(T_{r}\right)$ and $s_{1}^{*}\left(T_{r}\right)$ in terms of well known fluid parameters. To this end we take into account the fact that the derivatives w.r.t. $T_{r}$ of the saturation entropies are related to the reduced molar heat capacities along the saturation curve via the well known relations

$$
\begin{aligned}
& \frac{\mathrm{d} s_{\mathrm{g}}^{*}}{\mathrm{~d} T_{\mathrm{r}}}=\frac{c_{\mathrm{sat}}^{* \mathrm{~g}}}{T_{\mathrm{r}}}, \\
& \frac{\mathrm{d} s_{1}^{*}}{\mathrm{~d} T_{\mathrm{r}}}=\frac{c_{\mathrm{sat}}^{* 1}}{T_{\mathrm{r}}},
\end{aligned}
$$

where $c_{\text {sat }}^{*}=c_{\text {sat }} / R, c_{\text {sat }}$ being the molar heat capacity along the saturation curve. Recently, ${ }^{1}$ we have shown that $\xi^{*}\left(T_{r}\right) \equiv c_{\mathrm{sat}}^{* \mathrm{~g}} / T_{r}$ attains a maximum value $\xi_{\mathrm{M}}^{*}$ at a point $\mathrm{M}$ with reduced 
temperature $T_{\mathrm{M} r} \approx 0.81$. The physical meaning of the point $\mathrm{M}$ becomes clear by noticing that those fluids with $\xi_{\mathrm{M}}^{*}<0$ have a negative slope for any temperature of the vapor saturated branch between the triple and the critical points and, consequently, they are 'wet' fluids. On the contrary, those fluids with $\xi_{\mathrm{M}}^{*}>0$ present a zone around the point $\mathrm{M}$ with positive slope for the vapor saturated branch, behaving as 'dry' fluids. Fluids are either wet or dry but those with $\left|\xi_{\mathrm{M}}^{*}\right|$ close to zero can be termed as 'isentropic'.

By considering only the saturated vapor branch, from eq 10 one has

$$
\xi^{*}\left(T_{r}\right) \equiv \frac{c_{\mathrm{sat}}^{* \mathrm{~g}}}{T_{r}}=-a-0.615 B(\omega) \frac{\left(1-0.62 T_{r}\right)}{T_{r}^{2}\left(1-T_{r}\right)^{0.62}}
$$

Then, taking point $\mathrm{M}$ as reference, eq 12 yields

$$
a=-\xi_{\mathrm{M}}^{*}-0.615 B(\omega) \frac{\left(1-0.62 T_{\mathrm{M} r}\right)}{T_{\mathrm{M} r}^{2}\left(1-T_{\mathrm{M} r}\right)^{0.62}},
$$

which provides the parameter $a$ in terms of $\omega, T_{\mathrm{Mr} r}$ and $\xi_{\mathrm{M}}^{*}$.

Equations 8 and 9 , with the parameter $a$ given by eq 13 , are the basis of the present work since they allow for obtaining extended corresponding states expressions for $s_{\mathrm{g}}^{*}\left(T_{r}\right)$ and $s_{1}^{*}\left(T_{r}\right)$. In what follows we shall refer to these results as approximation A1 and denote them by $s_{\mathrm{g}, \mathrm{A} 1}^{*}\left(T_{r}\right)$ and $s_{1, \mathrm{~A} 1}^{*}\left(T_{r}\right)$, respectively. The values of $T_{\mathrm{M} r}$ and $\xi_{\mathrm{M}}^{*}$ required by approximation $A 1$ can be readily obtained from RefProp 9.1 (see ref 1 ) and are listed in Table S1 included in the Supporting Information associated with this article.

But, what about fluids for which $T_{\mathrm{M} r}$ and $\xi_{\mathrm{M}}^{*}$ are not available? Recently, ${ }^{1}$ we have shown that both $T_{\mathrm{M} r}$ and $\xi_{\mathrm{M}}^{*}$ present a fairly good correlation with the critical molar volume $v_{c}$. In particular, $T_{\mathrm{M} r}$ lie into the reduced temperature range $0.79-0.83$ with a mean value of $T_{\mathrm{M} r} \approx 0.81$, while $\xi_{\mathrm{M}}^{*}$ is correlated with the intermolecular separation at zero potential energy, $\sigma_{c}=\left(0.317 v_{c} / N_{A}\right)^{1 / 3}$ (being $N_{A}$ the Avogadro number), via the cubic equation:

$$
\xi_{\mathrm{M}}^{*}\left(\sigma_{c}\right)=40.4747-295.354\left(\sigma_{c} / \mathrm{nm}\right)+465.566\left(\sigma_{c} / \mathrm{nm}\right)^{2}-57.8077\left(\sigma_{c} / \mathrm{nm}\right)^{3} .
$$


Then, using $T_{\mathrm{M} r}=0.81$ into eq 13 , the slope $a$ can be approximated by

$$
a \approx-\xi_{\mathrm{M}}^{*}\left(\sigma_{c}\right)-1.3066 B(\omega),
$$

with $B(\omega)$ and $\xi_{\mathrm{M}}^{*}\left(\sigma_{c}\right)$ given by eqs 7 and 14 , respectively. The values of $\sigma_{c}$ for the 121 fluids considered in the RefProp 9.1 program are listed in Table S1, these data have been obtained from the corresponding molar critical volumes provided by RefProp 9.1.

The approximate results obtained from eqs 8,9 , and 5 , with $a$ given by eq 15 shall be referred to as approximation $\mathrm{A} 2$ and denoted by $s_{\mathrm{g}, \mathrm{A} 2}^{*}, s_{1, \mathrm{~A} 2}^{*}$, and $s_{0.385, \mathrm{~A} 2}^{*}$, respectively.

\section{Results and Discussion}

Figure 2 shows the liquid-vapor saturation curve in a $T_{r}-s^{*}$ diagram for R32, RE143a and pentane. The symbols correspond to results obtained from the RefProp 9.1 program $^{5}$ for $s_{\mathrm{g}}^{*}, s_{1}^{*}$, and $s_{x}^{*}$ (obtained from eq 1 with $x=0.385$ ). In what follows, these RefProp 9.1 results shall be denoted as $s_{\mathrm{g}, \mathrm{RP}}^{*}\left(T_{r}\right), s_{1, \mathrm{RP}}^{*}\left(T_{r}\right)$, and $s_{0.385, \mathrm{RP}}^{*}\left(T_{r}\right)$, respectively. The lines correspond to the approximate results $s_{\mathrm{g}, \mathrm{A} 1}^{*}$ and $s_{1, \mathrm{~A} 1}^{*}$, and $s_{0.385, \mathrm{~A} 1}^{*}$ obtained from eqs 8,9 , and 5, with $a$ given by eq 13 , using the following values: $\omega=0.2769, T_{\mathrm{M} r}=0.8198$ and $\xi_{\mathrm{M}}^{*}=-7.7684$ for R32, a wet fluid; $\omega=0.289, T_{\mathrm{M} r}=0.8180$ and $\xi_{\mathrm{M}}^{*}=-0.1089$ for RE143a, an isentropic fluid; and $\omega=0.251, T_{\mathrm{M} r}=0.8160$ and $\xi_{\mathrm{M}}^{*}=8.4366$ for pentane, a dry fluid. The parameters $\omega, T_{\mathrm{M} r}$ and $\xi_{\mathrm{M}}^{*}$ for the 121 fluids considered by the NIST program RefProp 9.1 are reported in ref 1 , and have also been listed in Table S1. For the three considered fluids we obtain an excellent agreement between RefProp 9.1 results and calculated data in the range $0.6<T_{r}<1$, but this agreement becomes slightly worse for R32 and pentane for temperatures below $T_{r}=0.6$. This fact can be attributed to the deviation of $s_{0.385, \mathrm{RP}}^{*}\left(T_{r}\right)$ from the linear behavior given by eq 5 , for $T_{r}<0.6$. In any case, the agreement obtained for R32 and pentane for $T_{r}<0.6$ is still rather good. This behavior is also obtained for

other fluids. Finally, we note that the reduced triple point temperature $T_{\operatorname{tp} r}=T_{\mathrm{tp}} / T_{c}$ lies in 

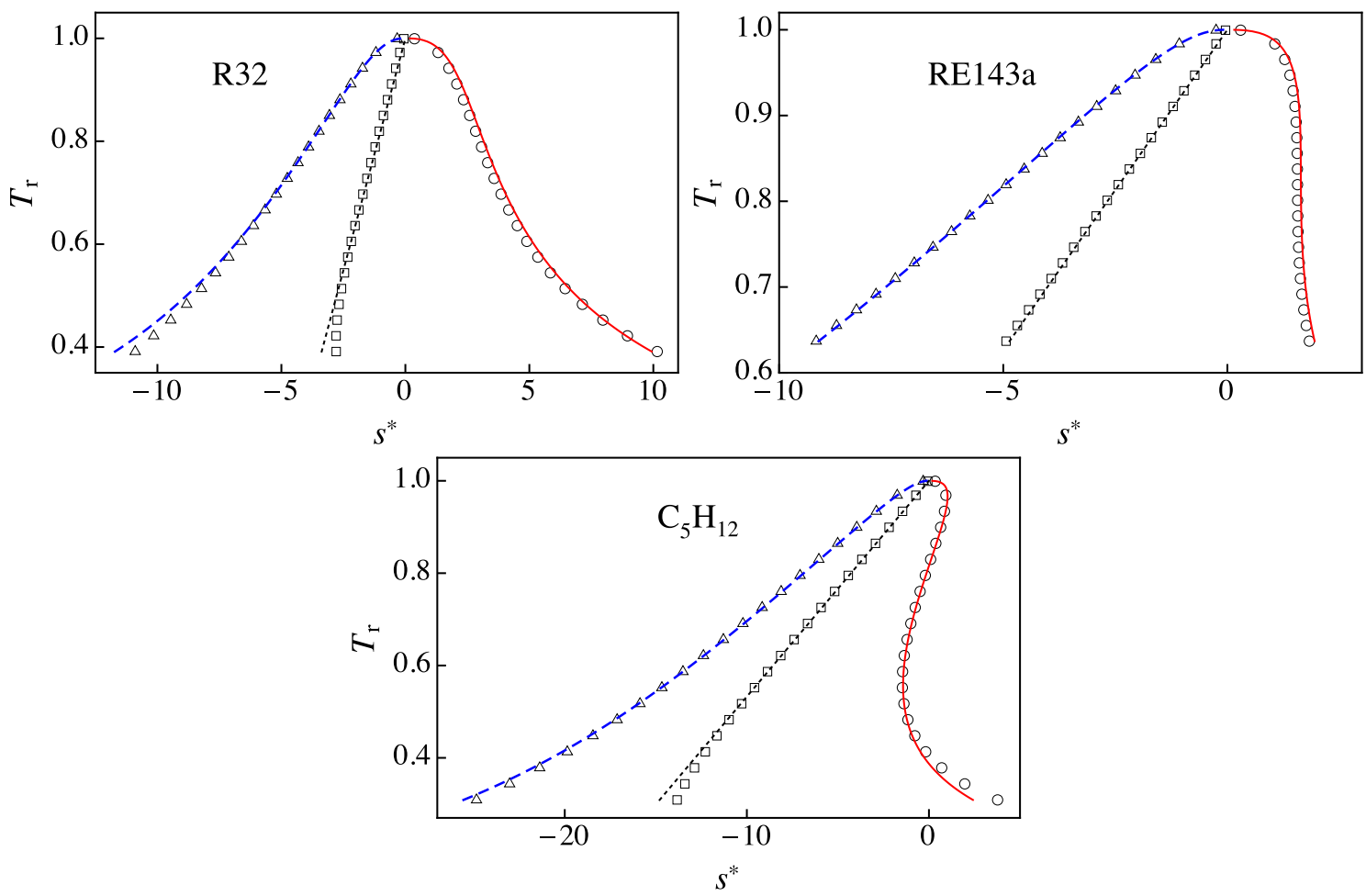

Figure 2: The liquid-vapor saturation curve in a $T_{\mathrm{r}}-s^{*}$ diagram for R32, RE143a and pentane. The lines are the approximate results $s_{\mathrm{l}, \mathrm{A} 1}^{*}$ (dashed blue lines), $s_{\mathrm{g}, \mathrm{A} 1}^{*}$ (red lines), and $s_{0.385, \mathrm{~A} 1}^{*}$ (dotted lines) obtained from eqs 9,8 , and 5 , respectively, with the parameter $a$ given by eq 13 (see text). The symbols are the RefProp $9.1^{5}$ results $s_{1, \mathrm{RP}}^{*}$ (triangles), $s_{\mathrm{g}, \mathrm{RP}}^{*}$ (circles), and $s_{0.385, \mathrm{RP}}^{*}$ (squares). $s_{0.385, \mathrm{RP}}^{*}$ has been obtained from eq 1

the range $0.3<T_{\operatorname{tp} r}<0.5$ for most pure fluids available in the RefProp 9.1 program. This indicates that using reduced temperatures in the range $0.4 \sim 0.6<T_{r}<1$ covers the most relevant fluid region in most applications involving phase coexistence.

In order to provide a quantitative measurement of the deviations of the approximate results $s_{\mathrm{g}, \mathrm{A} 1}^{*}$ and $s_{1, \mathrm{~A} 1}^{*}$ it is advisable to introduce the following definition for the area of the absolute deviation of the liquid-vapor curve in the $s^{*}-T_{\mathrm{r}}$ diagram, for $T_{r}>0.6$ :

$$
\Delta_{A 1}=\int_{0.6}^{1}\left|s_{\mathrm{g}, \mathrm{RP}}^{*}\left(T_{r}\right)-s_{\mathrm{g}, \mathrm{A} 1}^{*}\left(T_{r}\right)\right| \mathrm{d} T_{r}+\int_{0.6}^{1}\left|s_{1, \mathrm{RP}}^{*}\left(T_{r}\right)-s_{1, \mathrm{~A} 1}^{*}\left(T_{r}\right)\right| \mathrm{d} T_{r}
$$

where, as previously mentioned, the label RP indicates RefProp 9.1 results. The shaded surface in Figure 3 shows the area measured by eq 16 for MM (Hexamethyldisiloxane). 


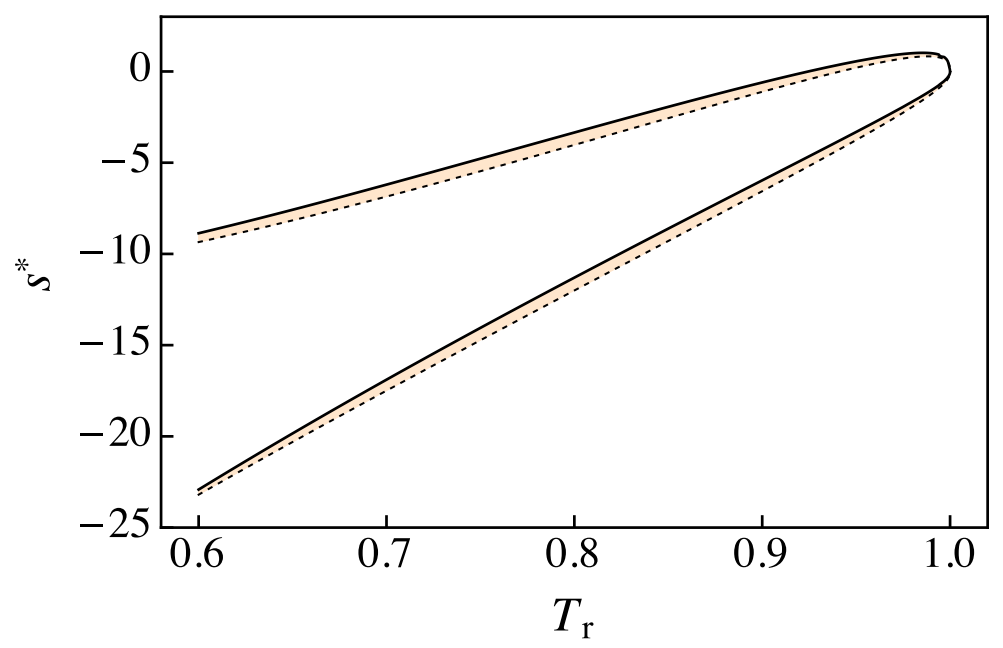

Figure 3: Absolute deviation of the liquid-vapor curve in a $s^{*}-T_{\mathrm{r}}$ diagram for MM. The dotted lines are the approximate results $s_{1, \mathrm{~A} 1}^{*}$ and $s_{\mathrm{g}, \mathrm{A} 1}^{*}$ obtained from eqs 9,8 , and 5 , respectively, with the parameter $a$ given by eq 13. The solid lines are the RefProp $9.1^{5}$ results $s_{1, \mathrm{RP}}^{*}$ and $s_{\mathrm{g}, \mathrm{RP}}^{*}$. The area of the shaded surface is determined by eq 16

Dividing $\Delta_{A 1}$ by the area of $s_{\mathrm{RP}}^{*}\left(T_{r}\right)$ and multiplying by 100 we obtain the percent relative deviation

$$
\Delta_{r 1}=100 \frac{\Delta_{A 1}}{\int_{0.6}^{1}\left|s_{\mathrm{g}, \mathrm{RP}}^{*}\left(T_{r}\right)-s_{1, \mathrm{RP}}^{*}\left(T_{r}\right)\right| \mathrm{d} T_{r}} .
$$

For the case of MM shown in Figure 3 we obtain $\Delta_{r 1}=13.84 \%$. Table S1 presents the percent relative deviation $\Delta_{r 1}$ for the 121 fluids considered in RefProp 9.1. For the fluids of Figure 2, R32, RE143a and pentane, we obtain $\Delta_{r 1}=3.34 \%, 1.71 \%$, and $1.33 \%$, respectively. These results are typical for most fluids although larger deviations are obtained for some fluids like R40 $\left(\Delta_{r 1}=12.65 \%\right)$, Helium $\left(\Delta_{r 1}=13.86 \%\right)$ and some fluids with large values of $\xi_{\mathrm{M}}^{*}$, i.e., for very dry fluids (see Table S1). The average percent relative deviation for all fluids is $\overline{\Delta_{r 1}}=5.00 \%$. The maximum percent relative deviation is obtained for D6 with a value $\Delta_{r 1, \max }=34.10 \% .96$ fluids out of 121 present a deviation $\Delta_{r 1}$ less than $5 \%$

Table S2 included in the Supporting Information associated with this article is an extension of the results of Figure 2 to all RefProp 9.1 pure fluids. Note, however, that for the sake of comparison we only include results in the range $0.6<T_{r}<1$. Overall, the observed behavior is similar to that of Figure 2. The important differences observed for Helium are 

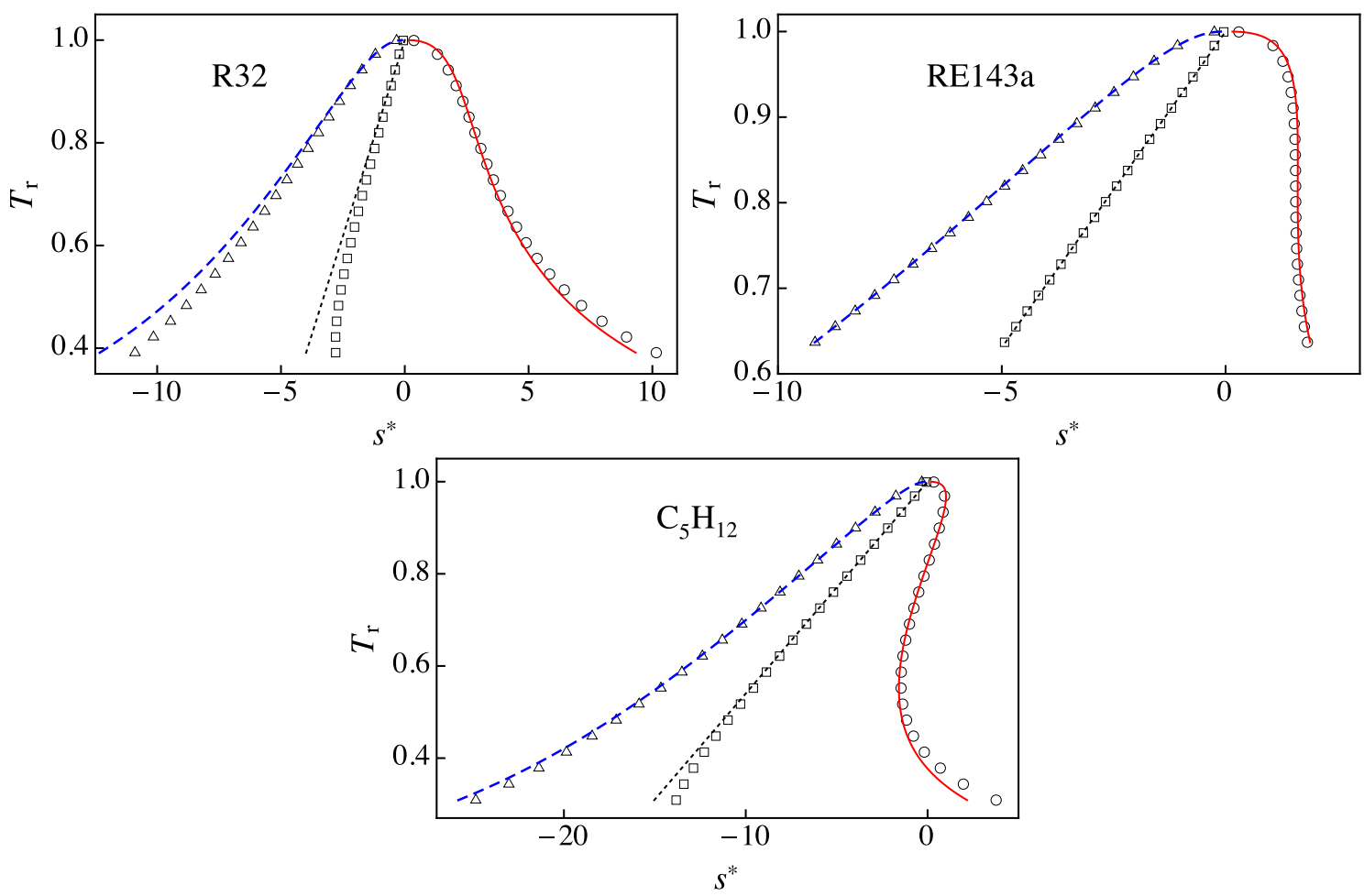

Figure 4: Same caption as in Figure 2 but now the lines are the approximate results $s_{1, \mathrm{~A} 2}^{*}$ (dashed blue lines), $s_{\mathrm{g}, \mathrm{A} 2}^{*}$ (red lines), and $s_{0.385, \mathrm{~A} 2}^{*}$ (dotted lines) with the parameter $a$ given by eq 15 (see text).

mainly due to the large deviation of $s_{0.385, \mathrm{~A} 1}^{*}\left(T_{r}\right)$ from the RefProp 9.1 result $s_{0.385, \mathrm{RP}}^{*}\left(T_{r}\right)$. The same behavior is observed for very dry fluids with large $\Delta_{r 1}$. In all cases, the differences between $s_{0.385, \mathrm{~A} 1}^{*}\left(T_{r}\right)$ and $s_{0.385, \mathrm{RP}}^{*}\left(T_{r}\right)$ are mainly related to a different inclination of the liquid-vapor curve in the $T_{\mathrm{r}}-s^{*}$ diagram. For some very dry fluids the (slightly) different inclination leads to large deviations $\Delta_{r 1}$ (see, e.g., the MM case of Figure 4). However we do not expect that the approximate results $s_{\mathrm{g}, \mathrm{A} 1}^{*}\left(T_{r}\right)$ and $s_{1, \mathrm{~A} 1}^{*}\left(T_{r}\right)$ would lead to large deviations when used, for instance, in calculating efficiencies of ORCs.

Like in the previous approximation $A 1$ we define the percent relative deviation of approximation $A 2$ as follows:

$$
\Delta_{r 2}=100 \frac{\Delta_{A 2}}{\int_{0.6}^{1}\left|s_{\mathrm{g}, \mathrm{RP}}^{*}\left(T_{r}\right)-s_{1, \mathrm{RP}}^{*}\left(T_{r}\right)\right| \mathrm{d} T_{r}} .
$$


where now

$$
\Delta_{A 2}=\int_{0.6}^{1}\left|s_{\mathrm{g}, \mathrm{RP}}^{*}\left(T_{r}\right)-s_{\mathrm{g}, \mathrm{A} 2}^{*}\left(T_{r}\right)\right| \mathrm{d} T_{r}+\int_{0.6}^{1}\left|s_{1, \mathrm{RP}}^{*}\left(T_{r}\right)-s_{1, \mathrm{~A} 2}^{*}\left(T_{r}\right)\right| \mathrm{d} T_{r}
$$

Figure 4 shows with lines the results for $s_{\mathrm{g}, \mathrm{A} 2}^{*}, s_{1, \mathrm{~A} 2}^{*}$, and $s_{0.385, \mathrm{~A} 2}^{*}$, in a $T_{r}-s^{*}$ diagram for R32 $\left(v_{c}=0.1227 \mathrm{~m}^{3} / \mathrm{kmol}, \sigma_{c}=0.4012 \mathrm{~nm}\right)$, RE143a $\left(v_{c}=0.2151 \mathrm{~m}^{3} / \mathrm{kmol}, \sigma_{c}=0.4838\right.$ $\mathrm{nm})$ and pentane $\left(v_{c}=0.311 \mathrm{~m}^{3} / \mathrm{kmol}, \sigma_{c}=0.5470 \mathrm{~nm}\right)$. Like in Figure 2 these approximate results are compared with RefProp $9.1^{5}$ results (symbols). The approximate results for $\operatorname{RE143a}\left(\Delta_{r 2}=1.47 \%\right)$ and pentane $\left(\Delta_{r 2}=1.27 \%\right)$ are rather good and show almost no variation compared to those presented in Figure 2. However, the approximate liquid-vapor saturation $T_{r}-s^{*}$ diagram for R32 shown in Figure 4 now present slightly larger deviations from the RefProp 9.1 results than the approximate diagram plotted in Figure 2, with a deviation $\Delta_{r 2}=5.01 \%$. This fact can be ascribed to the correlation eq 14 that yields worse predictions for R32 than for RE143a and pentane. Furthermore, we have checked that the proposed liquid-vapor saturation entropies curves change little for small changes of the parameters $x$ and $T_{\mathrm{Mr} r}$, but they are very sensitive to changes in the parameter $\xi_{\mathrm{M}}^{*}$. In any case, we remark that these saturation entropy curves only need as input data the acentric factor $\omega$ and the molar critical volume $v_{c}$.

Table S3 included in the Supporting Information extends the results of Figure 4 to all pure fluids considered by RefProp 9.1. The label for each plot in Table S3 includes the parameters required by eqs 8,9 , and 15 that are also listed in Table S1. In many cases (like RE143a and pentane), the predictions of eq 15 for the slope $a$ are very good and the approximate results plotted in Table S3 show excellent agreement with the RefProp 9.1 data for the $T_{r}-s^{*}$ saturation boundary. There are other cases where relevant discrepancies are observed. In these cases the approximation eq 15 with $\xi_{\mathrm{M}}^{*}$ given by the correlation eq 14 fails to yield an accurate result for the slope $a$. This failure is due to the differences between observed results for $\xi_{\mathrm{M}}^{*}$ and the predictions of 14 . The predictions for $a$ are very poor for the 
so-called quantum fluids (hydrogen, orthohydrogen, parahydrogen, deuterium and helium), and the siloxanes with large $v_{c}$ values (D4, D5, D6, MDM, MD2M, MD3M, and MD4M). These fluids where identified as oddball fluids in the derivation of eq 14 in ref 1.

Table S1 lists the percent relative deviation $\Delta_{r 2}$ for all fluids. In this case the average percent relative deviation is $\overline{\Delta_{r 2}}=14.54 \%$. The maximum percent relative deviation is obtained for D5 with a value $\Delta_{r 2, \max }=111.07 \% .93$ fluids out of 121 present a deviation $\Delta_{r 2}$ less than $15 \%$, among them 46 fluids have $\Delta_{r 2}<5 \%$.

\section{Summary}

To conclude, in this work we have presented an approximate expression for the liquid-vapor saturation curve in a $T_{r}-s^{*}$ diagram within an extended corresponding states scheme that only requires the knowledge of two well known parameters of the fluid: The critical molar volume $v_{c}$ and the acentric factor $\omega$. The derivation of this expression is based on the observation that for most fluids the line of constant quality $x$ in the liquid-vapor coexistence region in a $T_{r}-s^{*}$ diagram becomes very close to a straight line with slope $-a$ in the range $0.6<T_{r}<1$ for $x \approx 0.385$. In addition to the approximate linear behavior for $s_{0.385}^{*}$, the present approach requires an approximate expression for the enthalpy of vaporization. For simplicity we have chosen an extended corresponding states version ${ }^{16}$ of the Watson equation for the enthalpy of vaporization. ${ }^{17}$ More accurate corresponding states expressions for the enthalpy of vaporization in terms of the acentric factor $\omega^{18}$ could be used at a cost of increasing the complexity of the calculations without an improvement of the results of significant relevance for the present work.

The parameter $a$ has been shown to depend both on $\omega$ and the coordinates $\left(T_{\mathrm{M} r}, \xi_{\mathrm{M}}^{*}\right)$ of the

point $\mathrm{M}$ where $\xi^{*}\left(T_{r}\right)=c_{\mathrm{sat}}^{* g} / T_{r}$ attains its maximum value. At this point two approximations have been explored: (i) the approximation $A 1$ that considers the values of $T_{\mathrm{Mr}}$ and $\xi_{\mathrm{M}}^{*}$ reported in ref 1 for the 121 fluids of the RefProp 9.1 program, ${ }^{5}$ or (ii) the approximation 
$A 2$ that takes $T_{\mathrm{Mr}}=0.81$ and considers eq 14 that correlates $\xi_{\mathrm{M}}^{*}$ with the critical molar volume $v_{c}$. The approximation $A 1$ yields better results as shown in Figure 2 and Tables S1 and $\mathrm{S} 2$ but requires the knowledge of the parameters $T_{\mathrm{M} r}$ and $\xi_{\mathrm{M}}^{*}$. Perhaps more interesting is the approximation $A 2$ since it provides an extended corresponding states expression for the shape of the $T-s$ saturation boundary at the cost of yielding slightly worse results (see Figure 4). The results of the approximation $A 2$ have been shown to be very poor for the so-called quantum fluids and some siloxanes due to the correlation eq 14 of $\xi_{\mathrm{M}}^{*}$ with $v_{c}$ that gives rise to important relative deviations in the prediction for the slope $a$ (see Tables S1 and S3).

Finally we would like to comment on the utility of approximation $A 2$ in the search of new working fluids for ORCs where a good estimation of the liquid-vapor coexistence curve in a $T-s$ diagram is of major interest. Of course, as mentioned above, one should be cautious with the results obtained for some families of fluids like the siloxanes and the quantum fluids.

\section{Acknowledgement}

We thank financial support by Junta de Castilla y León of Spain under Grant SA017P17.

\section{Associated Content}

Supporting Information. Tables S1, S2, and S3. This material is available free of charge via the Internet at http://pubs.acs.org.

\section{References}

(1) White, J.; Velasco, S. Characterizing wet and dry fluids in temperature-entropy diagrams. Energy 2018, 154, $269-276$. 
(2) Bao, J.; Zhao, L. A review of working fluid and expander selections for organic Rankine cycle. Renew. Sust. Energ. Rev. 2013, 24, 325 - 342.

(3) Hærvig, J.; Sørensen, K.; Condra, T. Guidelines for optimal selection of working fluid for an organic Rankine cycle in relation to waste heat recovery. Energy 2016, 96, 592 $-602$.

(4) Györke, G.; Deiters, U. K.; Groniewsky, A.; Lassu, I.; Imre, A. R. Novel classification of pure working fluids for Organic Rankine Cycle. Energy 2018, 145, 288 - 300.

(5) Lemmon, E. W.; Huber, M. L.; McLinden, M. O. NIST Standard Reference Database 23: Reference Fluid Thermodynamic and Transport Properties-REFPROP, Version 9.1. National Institute of Standards and Technology, Standard Reference Data Program, Gaithersburg, 2013.

(6) Bell, I. H.; Wronski, J.; Quoilin, S.; Lemort, V. Pure and Pseudo-pure Fluid Thermophysical Property Evaluation and the Open-Source Thermophysical Property Library CoolProp. Ind. Eng. Chem. Res. 2014, 53, 2498-2508.

(7) Morrison, G. The shape of the temperature-entropy saturation boundary. Int. J. Refrig 1994, 17, $494-504$.

(8) Garrido, J. M.; Quinteros-Lama, H.; Mejía, A.; Wisniak, J.; Segura, H. A rigorous approach for predicting the slope and curvature of the temperature-entropy saturation boundary of pure fluids. Energy 2012, 45, 888 - 899, The 24th International Conference on Efficiency, Cost, Optimization, Simulation and Environmental Impact of Energy, ECOS 2011.

(9) Albornoz, J.; Mejía, A.; Quinteros-Lama, H.; Garrido, J. M. A rigorous and accurate approach for predicting the wet-to-dry transition for working mixtures in organic Rankine cycles. Energy 2018, 156, 509 - 519. 
(10) Groniewsky, A.; Györke, G.; Imre, A. R. Description of wet-to-dry transition in model ORC working fluids. Appl. Therm. Eng. 2017, 125, 963 - 971.

(11) Groniewsky, A.; Imre, A. R. Prediction of the ORC Working Fluid's TemperatureEntropy Saturation Boundary Using Redlich-Kwong Equation of State. Entropy 2018, 20, 93.

(12) Su, W.; Zhao, L.; Deng, S. New knowledge on the temperature-entropy saturation boundary slope of working fluids. Energy 2017, 119, 211 - 217.

(13) Su, W.; Zhao, L.; Deng, S.; Zhao, Y. How to predict the vapor slope of temperatureentropy saturation boundary of working fluids from molecular groups? Energy 2017, $135,14-22$.

(14) Bulavin, L.; Kulinskii, V.; Malomuzh, N. Peculiarities in the behavior of the entropy diameter for molecular liquids as the reflection of molecular rotations and the excluded volume effects. J. Mol. Liq. 2011, 161, $19-29$.

(15) Imre, A. R.; Quiñones-Cisneros, S. E.; Deiters, U. K. Adiabatic processes in the liquidvapor two-phase region. 1. Pure fluids. Ind. Eng. Chem. Res. 2014, 53, 13529-13542.

(16) Velasco, S.; Santos, M. J.; White, J. A. Extended corresponding states expressions for the changes in enthalpy, compressibility factor and constant-volume heat capacity at vaporization. J. Chem. Thermodyn. 2015, 85, 68-76.

(17) Watson, K. Prediction of critical temperatures and heats of vaporization. Ind. Eng. Chem. 1931, 23, 360-364.

(18) Mulero, A.; Cachadina, I.; Parra, M. I. Comparison of corresponding-states-based correlations for the prediction of the vaporization enthalpy of fluids. Ind. Eng. Chem. Res. 2008, $47,7903-7916$. 
TOC Graphic

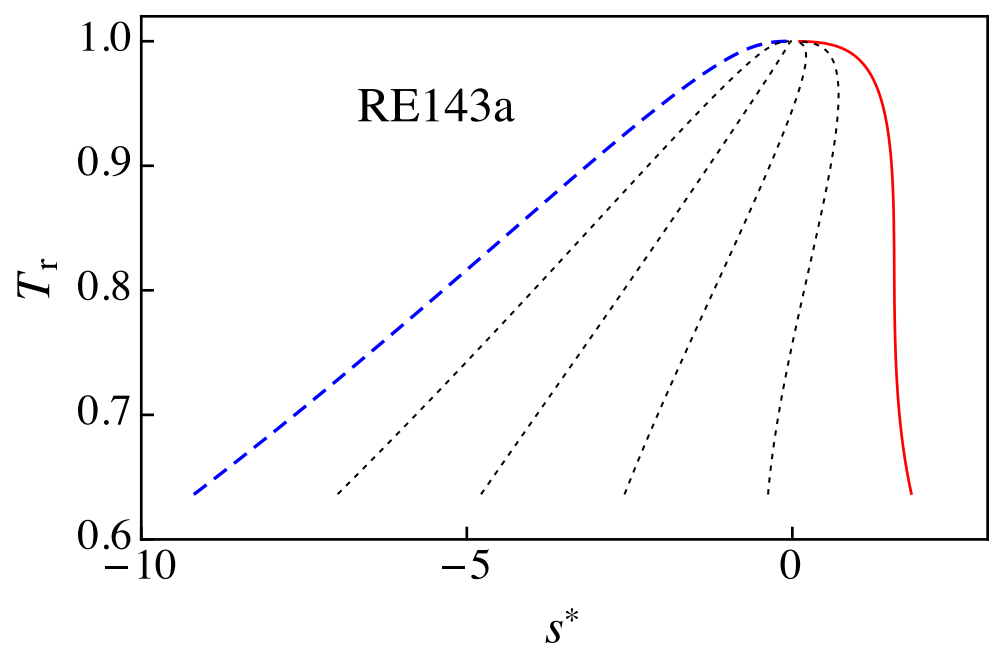

